\title{
ACEPTABILIDAD DEL CASTIGO FÍSICO EN LA CRIANZA DE LOS NIÑOS EN PERSONAS QUE FUERON VÍCTIMAS DE VIOLENCIA FÍSICA EN LA NIÑEZ EN PERÚ
}

\author{
Alejandra Burela ${ }^{1, a}$, Marina Piazza ${ }^{1,2, b}$, German F. Alvarado ${ }^{2, c}$, Alfonso Gushiken ${ }^{2, d}$, Fabián Fiestas $^{1, c}$
}

\begin{abstract}
RESUMEN
Objetivos. El estudio evaluó la asociación entre haber sido víctima de violencia física en la niñez y la aceptabilidad, en etapas posteriores de vida, hacia el uso del castigo físico en la crianza de los niños. Materiales y métodos. Se realizó un análisis secundario de un estudio sobre violencia en 6399 personas mayores de 14 años residentes de las ciudades de Lima, Callao, Maynas, Arequipa, Cusco, Trujillo y Huamanga. Se usó modelos de regresión logística univariados y multivariados para estimar asociaciones estadísticas. Resultados. La aceptabilidad del uso del castigo físico en la crianza de niños es mayor en personas con el antecedente de ser víctimas de violencia física durante la niñez en comparación con las no victimizadas (OR=1,8; IC 95\%: 1,5-2,1; $p<0,001)$ ajustando por potenciales variables de confusión. Conclusiones. Las personas expuestas a violencia física durante la niñez tienen más riesgo de aceptar o justificar la misma durante la adultez, lo que podría contribuir a mantener esta práctica de crianza de una generación a la siguiente. Iniciativas dirigidas a prevenir el uso del castigo físico en la crianza de niños se deben implementar para reducir la tendencia a reproducir el ejercicio de violencia en personas victimizadas.
\end{abstract}

Palabras clave: Violencia; Castigo; Maltrato a los Niños; Niños (fuente MeSH NLM).

\section{ACCEPTABILITY OF PHYSICAL PUNISHMENT IN CHILD REARING BY PEOPLE WHO WERE VICTIMS OF PHYSICAL VIOLENCE DURING CHILDHOOD IN PERU}

\begin{abstract}
Objectives. This study evaluated the association between having been a victim of physical violence during childhood and the acceptability, in later life, towards the use of physical punishment in child rearing. Materials and methods. A secondary analysis was conducted of a study on violence in 6,399 people over 14 years of age living in the cities of Lima, Callao, Maynas, Arequipa, Cusco, Trujillo and Huamanga. Univariate and multivariate logistic regression models were used to estimate statistical associations. Results. The acceptability of the use of physical punishment in child rearing is higher in people who were victims of physical abuse during childhood compared with non-victimized people $(\mathrm{OR}=1.8$; $95 \% \mathrm{Cl}: 1.5-21 ; \mathrm{p}<0.001)$ after adjusting for potential confounders. Conclusions. People exposed to physical violence during childhood are more likely to accept or justify violence in adulthood, which could help maintain this child rearing practice from one generation to the next. Initiatives aimed at preventing the use of physical punishment in child rearing should be implemented to reduce the tendency to reproduce the action of violence by victimized people.
\end{abstract}

Key words: Violence; Punishment; Child abuse; Child (source MeSH NLM).

\section{INTRODUCCIÓN}

De acuerdo con la Convención sobre los Derechos del Niño, los niños tienen derecho a no ser objeto de ninguna forma de violencia (1). Esta convención internacional define castigo físico como: "Todo castigo en el que se utilice la fuerza física y que tenga por objeto causar cierto grado de dolor o malestar, aunque sea leve". Por lo general, incluye prácticas de pegar a los niños ("manotazos", "bofetadas", "palizas"), con la mano o con algún objeto (palo, cinturón, zapato, cuchara de madera, u otro objeto). Otras formas de castigo físico consideradas son: dar puntapiés, sacudir o empujar a los niños, arañarlos, pellizcarlos, morderlos, tirarles del pelo o de las orejas, obligarlos a ponerse en posturas incómodas o producirles quemaduras ${ }^{(1)}$.

\footnotetext{
Unidad de Análisis y Generación de Evidencias en Salud Pública (UNAGESP), Instituto Nacional de Salud, Lima, Perú.

Facultad de Salud Pública y Administración, Universidad Peruana Cayetano Heredia. Lima, Perú.

Bióloga epidemióloga; ${ }^{\mathrm{b}}$ psicóloga epidemióloga; ${ }^{\mathrm{c}}$ médico epidemiólogo; ${ }^{\mathrm{d}}$ médico salubrista.

Recibido: : 15-04-14 Aprobado: 05-11-14
} 
En el mundo, en promedio, seis de cada diez niños de dos a catorce años de edad, sufren de manera periódica castigos físicos por parte de sus cuidadores ${ }^{(2)}$. En Chile, el $53,9 \%$ de los niños recibe castigo físico ${ }^{(3)}$. En familias rurales en Colombia, $41 \%$ de los padres admitieron usar el castigo físico como medida disciplinaria ${ }^{(4)}$.

La violencia contra los niños en el hogar es frecuente en el Perú (5). En el 2008, la Encuesta Nacional de Salud Familiar-Varones, evidenció que el $52,5 \%$ de padres y madres recurren a violencia física para corregir a los niños ${ }^{(6)}$. A esta extensa frecuencia con que se da la violencia contra niños en el Perú, se añade evidencia que hasta el $50 \%$ del maltrato infantil ocurre antes de los seis años de edad ${ }^{(7)}$. Esto genera, además, una enorme preocupación sobre el número de personas que podría vivir con secuelas psicológicas y físicas como consecuencia de la violencia sufrida durante la infancia y que a largo plazo puede generar trastornos en el neurodesarrollo y problemas de salud mental en la vida adulta como depresión y abuso de sustancias psicoactivas, comportamiento criminal y antisocial y, por último, violencia doméstica ${ }^{(8,9)}$. De igual manera, existe evidencia del impacto de haber sufrido violencia durante la infancia y dificultades académicas ${ }^{(10)}$ deserción escolar ${ }^{(11)}$ y menores expectativas educativas ${ }^{(12)}$.

Si bien no todos los niños que han sido víctimas de violencia se vuelven perpetradores de esta ${ }^{(13)}$, la probabilidad de ejercerla se incrementa ${ }^{(14-16)}$. Existe evidencia de que personas que han sido víctimas de violencia física en la infancia, en la vida adulta tienen un riesgo 11,6 veces mayor de ejercer este tipo de violencia, en comparación con personas que no han sido víctimas ${ }^{(17)}$. De igual forma, un estudio realizado en Perú sobre violencia transgeneracional en madres peruanas, reportó que el $36,1 \%$ de mujeres que han sido golpeadas de niñas, golpean a sus hijos ${ }^{(18)}$. Adicionalmente, la violencia infantil también se asocia con otros tipos de violencia doméstica en la adultez. Así, un estudio en población general peruana de Fiestas et al., encontró que el haber sido víctima de violencia física en la niñez estuvo asociado con mayor riesgo de violencia de pareja en la edad adulta ${ }^{(19)}$.

Por otro lado, se ha reportado que en adultos, la aceptabilidad o justificación del castigo físico en la crianza de los niños se asocia con el empleo de la violencia física contra ellos ${ }^{(20,21)}$. Según la teoría de aprendizaje social, la transmisión transgeneracional de un patrón de crianza puede explicarse debido al aprendizaje del comportamiento en el ambiente familiar. Así, el niño victimizado aprende que el castigo físico es una forma de corrección aceptable (22). Esto, a su vez, es consistente con la teoría del procesamiento de información social, la cual sostiene que los procesos cognitivos de los padres influyen en la decisión de ejercer violencia física hacia los hijos ${ }^{(23)}$.
Así pues, la aceptabilidad o justificación del castigo físico parece formar parte del proceso que ocurre entre haber sido expuesto a violencia en la infancia y el ejercerla de manera activa en la vida adulta. Alguna evidencia se ha encontrado en favor de esta hipótesis. Así, un estudio de Orué et al., encontró que la aceptabilidad del castigo físico actuó como mediador entre la exposición indirecta a la violencia física y la conducta agresiva en la infancia (24). Otro estudio realizado por Calvete et al., encontró que la aceptabilidad del castigo físico también tuvo un rol mediador entre haber sido víctima de castigo en la infancia y presentar problemas de conducta en la adolescencia ${ }^{(25)}$. No se ha encontrado evidencia en el contexto sociocultural peruano, de frecuente práctica de castigo físico a niños en el hogar, de que este tipo de violencia se relacione con su aceptabilidad en etapas posteriores de la vida, incluyendo la etapa de adultez del individuo y, por lo tanto, lo coloque con una mayor probabilidad de ejercer violencia contra niños.

Así, el objetivo principal del presente estudio fue evaluar si existe, en una muestra de población general peruana, evidencia consistente con la hipótesis de que la exposición a violencia física en la niñez está asociada con un mayor riesgo de que la persona en una etapa posterior de la vida considere al castigo físico infantil como una conducta aceptable. Dada la evidencia de que la aceptabilidad del empleo del castigo físico está asociada a la perpetración de actos violentos contra niños, la información generada localmente puede ser relevante para el diseño de estrategias de prevención de la violencia física contra niños en el Perú.

\section{MATERIALES Y MÉTODOS}

Se realizó un análisis secundario de la base de datos de la Encuesta Poblacional de Creencias, Actitudes y Prácticas sobre la Violencia aplicada por el Instituto Cuánto, por encargo del Ministerio del Interior, en 2006, en siete provincias del Perú: Lima, Callao, Maynas, Arequipa, Cusco, Trujillo y Huamanga. La muestra incluyó 6399 sujetos a partir de los 15 años de edad. El diseño de muestreo fue estratificado y trietápico ${ }^{(19)}$. Teniendo como estratos a las provincias, la primera unidad de muestreo fueron las manzanas, la segunda unidad las viviendas y la tercera unidad, los individuos seleccionados por vivienda. Se seleccionaron 400 individuos por cada uno de los siete distritos de Lima Metropolitana y 400 por cada provincia fuera de Lima.

Se utilizó el cuestionario de violencia desarrollado y validado por el proyecto ACTIVA de la Organización Panamericana de la Salud (26). La variable "exposición a castigo físico en la infancia" se midió con la pregunta: 
cuando usted era niño y hacía una «travesura» o «desobediencia», ¿cómo lo corregían? Todas las respuestas fueron dicotomizadas en "sí" (valor de 1) y "no" (valor 0) a castigo físico. Se consideró "sí" a aquellas respuestas que involucraron contacto físico (e.g. patada, jalón de pelos, puñetazos, golpes con objetos, entre otras acciones de acuerdo con la definición de castigo físico presentada en la introducción. Se consideró "No" a todas las otras posibles respuestas (e.g. grito, encierro, no corrección de ningún tipo).

Posteriormente a la pregunta del antecedente de haber sido víctima durante la niñez de castigo físico, se evalúa la aceptabilidad o justificación del castigo físico en la crianza de los niños a través de la pregunta: ¿es a veces necesario el castigo físico para educar a los niños? La respuesta de la escala Likert se categorizó como variable dicotómica con valor de 1 (Sí) cuando la respuesta fue "totalmente de acuerdo" o "de acuerdo". Las respuestas que incluyen "no seguro", "en desacuerdo" y "totalmente en desacuerdo" se categorizaron con el valor de 0 ("no/ no seguro").

Otras variables consideradas en el modelo como potencialmente confusoras fueron: edad, sexo, provincia de residencia, grado de instrucción, ingreso familiar mensual promedio y consumo de alcohol excesivo en el último mes (consumo excesivo: ocho vasos de cerveza o cinco vasos de otro licor en una misma ocasión).

Se realizó un análisis univariado para obtener las frecuencias y las proporciones de las variables de interés en el estudio. Luego se realizó un análisis bivariado, utilizando la prueba de chi cuadrado para observar la distribución de estas variables de interés según la variable desenlace: aceptabilidad del castigo en la crianza de los niños. Por último, se utilizó regresión logística univariada y luego múltiple para estimar los odds ratios (ORs) de asociación entre la aceptabilidad del castigo físico en la crianza de los niños y el antecedente de exposición a violencia física en la infancia ajustando por las variables potencialmente confusoras incluidas en el modelo. El estudio tuvo un diseño de muestreo complejo. Sin embargo, no se pudo tener acceso a la información referente a dicho diseño ni a la probabilidad de selección. Por lo tanto, se realizó un modelamiento para muestras aleatorias simples con una varianza robusta, utilizando la unidad primeria de muestreo (UPM) como cluster. Se evaluaron los supuestos para los análisis bivariados y de múltiples variables, tomando en cuenta la limitación de observaciones independientes y tratando de corregirlo al trabajar con una varianza robusta. El análisis estadístico se realizó con el programa Stata versión 12.0.
Es posible que algunos lectores pueden encontrar que la construcción de la variable de aceptabilidad del castigo físico en la crianza de los niños debió haber sido construida de tal manera que aquellos que respondieron "no Seguro" deba ser incluido en el grupo de aquellos que respondieron "sí" (ie., "totalmente de acuerdo" y "de acuerdo"). Por ello, se realizó un análisis de sensibilidad categorizando la variable como 1 si la respuesta fue "sí/no sabe" y como 0 si la persona respondió "no" ("desacuerdo" y "totalmente en desacuerdo"). De igual forma, se realizó un segundo análisis de sensibilidad incluyendo solo a población a partir de 18 años, para así descartar la posibilidad de distorsionar el análisis al incluir en la población de estudio a adolescentes de 15 a 17 años ya que algunos podrían aún estar siendo víctimas de violencia doméstica.

Asimismo, otro análisis de sensibilidad tiene que ver con la controversia con respecto al uso de OR en vez de razones de prevalencias (RP) en estudios con diseño transversal. Así, se realizó también una regresión de Poisson para estimar RP.

La ejecución de la encuesta contó con la aprobación del Ministerio del Interior y fue supervisado por la Secretaría Técnica del Consejo Nacional de Seguridad Ciudadana (CONASEC). Se solicitó consentimiento verbal a los participantes del estudio. A partir de la existencia de la base de datos, el equipo de investigadores respondió una pregunta científica. Los investigadores no tuvieron acceso a códigos de identificación de los participantes.

\section{RESULTADOS}

Del total de la muestra de 6399 sujetos, el 15\% reportó considerar aceptable el castigo físico como recurso en la crianza de los niños. Por otro lado, 64\% reportaron haber sido víctimas de violencia física en algún momento de su niñez. En la Tabla 1 se presentan las distribuciones de cada una de las covariables con respecto a la aceptabilidad del castigo físico.

En la Tabla 2 se presenta el modelo crudo de regresión logística en el que se observa que la aceptación del castigo físico en la crianza de los niños fue más frecuente en personas que han sido víctimas de violencia física en la niñez en comparación con las que no la experimentaron (OR 2.0; IC 95\%: 1,7-2,3; p<0,001). La asociación entre aceptabilidad del castigo físico en la crianza de los niños y haber sido víctima de violencia física en la infancia siguió siendo significativa de manera robusta luego de ajustar por las variables potencialmente confusoras como edad, sexo, provincia de residencia, grado de instrucción, ingreso familiar mensual promedio y consumo de alcohol 
Tabla 1. Distribución de características de la muestra por aceptabilidad de castigo físico. Encuesta Poblacional de Creencias, Actitudes y Prácticas sobre la Violencia Perú, 2006

\begin{tabular}{|c|c|c|c|}
\hline & $\begin{array}{l}\text { Aceptabilidad } \\
\text { del castigo físico }\end{array}$ & $\begin{array}{l}\text { No aceptabilidad } \\
\text { de castigo físico }\end{array}$ & \\
\hline Características & $\mathrm{n}=960(\%)$ & $\mathrm{n}=5439(\%)$ & $p^{\dagger}$ \\
\hline \multicolumn{4}{|c|}{ Víctima de violencia física en niñez } \\
\hline No & $227(9,9)$ & $2058(90,1)$ & $<0,001$ \\
\hline Sí & $733(17,9)$ & $3366(82,1)$ & \\
\hline \multicolumn{4}{|l|}{ Edad en años } \\
\hline $15-17$ & $43(14,1)$ & $261(85,9)$ & 0,013 \\
\hline $18-29$ & $188(12,3)$ & $335(87,7)$ & \\
\hline $30-45$ & $381(15,7)$ & $2054(84,4)$ & \\
\hline 46-59 & $226(16,3)$ & $1164(83,7)$ & \\
\hline$>60$ & $121(16,7)$ & $603(83,3)$ & \\
\hline \multicolumn{4}{|c|}{ Grado de instrucción } \\
\hline $\begin{array}{l}\text { Secundaria } \\
\text { incompleta o menos }\end{array}$ & $316(17,4)$ & $1498(82,6)$ & 0,002 \\
\hline $\begin{array}{l}\text { Secundaria } \\
\text { completa }\end{array}$ & $336(15,6)$ & $1824(84,4)$ & \\
\hline $\begin{array}{l}\text { Universitaria o } \\
\text { superior no } \\
\text { universitaria completa }\end{array}$ & $90(12,0)$ & $663(88,1)$ & \\
\hline $\begin{array}{l}\text { Superior no } \\
\text { universitaria } \\
\text { completa }\end{array}$ & $98(13,0)$ & $655(87,0)$ & \\
\hline $\begin{array}{l}\text { Universitaria } \\
\text { completa }\end{array}$ & $120(13,0)$ & $799(86,9)$ & \\
\hline \multicolumn{4}{|l|}{ Sexo } \\
\hline Femenino & $531(14,0)$ & $3276(86,1)$ & 0,004 \\
\hline Masculino & $429(16,6)$ & $2163(83,5)$ & \\
\hline \multicolumn{4}{|c|}{ Consumo excesivo de alcohol en el último mes } \\
\hline Nunca & $525(14,3)$ & $3135(85,7)$ & 0,114 \\
\hline Una vez & $302(15,2)$ & $1691(84,9)$ & \\
\hline 304 veces & $112(17,8)$ & $518(82,2)$ & \\
\hline 5 a más veces & $21(18,1)$ & $958(1,9)$ & \\
\hline \multicolumn{4}{|c|}{ Ingreso familiar mensual promedio } \\
\hline Menos de 840 soles & $470(16,2)$ & $2432(83,8)$ & 0,050 \\
\hline $\begin{array}{l}\text { Entre } 840 \text { y menos } \\
\text { de } 1200\end{array}$ & $270(13,7)$ & $1695(86,3)$ & \\
\hline $\begin{array}{l}\text { Entre } 1200 \text { y } \\
\text { menos de } 2000\end{array}$ & $152(15,2)$ & $851(84,9)$ & \\
\hline Más de 2000 soles & $68(12,9)$ & $461(87,2)$ & \\
\hline \multicolumn{4}{|l|}{ Provincia } \\
\hline Lima & $556(14,5)$ & $3268(85,5)$ & $<0,001$ \\
\hline Callao & $59(14,4)$ & $350(85,6)$ & \\
\hline Trujillo & $79(18,1)$ & $358(81,9)$ & \\
\hline Arequipa & $49(11,5)$ & $378(88,5)$ & \\
\hline Cuzco & $54(12,8)$ & $368(87,2)$ & \\
\hline Huamanga & $91(22,6)$ & $311(77,4)$ & \\
\hline Maynas & $72(15,1)$ & $406(84,9)$ & \\
\hline
\end{tabular}

† prueba de chi2 (se tomó en cuenta valores p obtenidos de regresión bivariada donde se consideró una varianza robusta)

excesivo en el último mes (OR 1,8; IC 95\%: 1,6-2,2; $p<0,001)$. Los OR obtenidos en la regresión de Poisson no resultaron distintos a los odds ratios obtenidos en la regresión logística.

Secundariamente, con relación al nivel de asociación observado para cada una de las variables del modelo ajustado, se observa menor aceptabilidad del castigo físico en la crianza de los niños en personas
Tabla 2. Modelo crudo y ajustado entre el antecedente de victimización y la aceptabilidad de castigo físico. Encuesta Poblacional de Creencias, Actitudes y Prácticas sobre la Violencia Perú, 2006

\begin{tabular}{|c|c|c|c|c|c|}
\hline & \multicolumn{3}{|c|}{ Modelo crudo } & \multicolumn{2}{|c|}{ Modelo ajustado* } \\
\hline & OR & IC 95\% & $p$ & OR IC 95\% & $p$ \\
\hline \multicolumn{6}{|c|}{ Víctima de violencia física en niñez } \\
\hline No & 1,0 & -- & -- & 1,0 & -- \\
\hline Sí & 2,0 & $1,7-2,3$ & $<0,001$ & $1,81,6-2,2$ & $<0,001$ \\
\hline \multicolumn{6}{|l|}{ Edad en años } \\
\hline $15-17$ & 1,0 & -- & -- & 1,0 & - \\
\hline $18-29$ & 0,9 & $0,6-1,2$ & 0,403 & $0,90,6-1,3$ & 0,503 \\
\hline $30-45$ & 1,1 & $0,8-1,6$ & 0,503 & $1,1 \quad 0,8-1,6$ & 0,650 \\
\hline $46-59$ & 1,2 & $0,8-1,7$ & 0,379 & $1,1 \quad 0,8-1,7$ & 0,484 \\
\hline$>60$ & 1,2 & $0,8-1,8$ & 0,322 & $1,2 \quad 0,8-1,7$ & 0,489 \\
\hline \multicolumn{6}{|l|}{ Grado de instrucción } \\
\hline $\begin{array}{l}\text { Secundaria incompleta o } \\
\text { menos }\end{array}$ & 1,0 & -- & -- & 1,0 & -- \\
\hline Secundaria completa & 0,9 & $0,7-1,0$ & 0,115 & $0,90,8-1,1$ & 0,490 \\
\hline $\begin{array}{l}\text { Universitaria o superior } \\
\text { no universitaria completa }\end{array}$ & 0,6 & $0,5-0,8$ & 0,001 & $-0,9$ & 0,020 \\
\hline $\begin{array}{l}\text { Superior no universitaria } \\
\text { completa }\end{array}$ & 0,7 & $0,5-0,9$ & 0,009 & $0,8 \quad 0,6-1,0$ & 0,050 \\
\hline Universitaria completa & 0,7 & $0,6-0,9$ & 0,004 & $0,8 \quad 0,6-1,0$ & 0,030 \\
\hline \multicolumn{6}{|l|}{ Sexo } \\
\hline Femenino & 1,0 & -- & -- & 1,0 & -- \\
\hline Masculino & 1,2 & $1,1-1,4$ & 0,004 & $1,21,0-1,4$ & 0,020 \\
\hline \multicolumn{6}{|c|}{ Consumo excesivo de alcohol en el último mes } \\
\hline Nunca & 1,0 & -- & -- & 1,0 & -- \\
\hline Una vez & 1,1 & $0,9-1,2$ & 0,400 & $1,0 \quad 0,9-1,2$ & 0,920 \\
\hline 3 ó 4 vece & 1,3 & $1,0-1,6$ & 0,043 & $1,21,0-1,6$ & 0,080 \\
\hline 5 a más veces & 1,3 & $0,8-2,1$ & 0,241 & $1,2 \quad 0,7-2,0$ & 0,441 \\
\hline \multicolumn{6}{|c|}{ Ingreso familiar mensual promedio } \\
\hline Menos de 840 soles & 1,0 & -- & -- & 1,0 & -- \\
\hline $\begin{array}{l}\text { Entre } 840 \text { y menos de } \\
1200\end{array}$ & 0,8 & $0,7-1,0$ & 0,030 & $0,90,8-1,1$ & 0,242 \\
\hline $\begin{array}{l}\text { Entre } 1200 \text { y menos de } \\
2000\end{array}$ & 0,9 & $0,7-1,2$ & 0,482 & $1,1 \quad 0,9-1,4$ & 0,500 \\
\hline Más de 2000 soles & 0,8 & $0,6-1,0$ & 0,071 & $1,0 \quad 0,7-1,4$ & 0,952 \\
\hline \multicolumn{6}{|l|}{ Provincia } \\
\hline Lima & 1,0 & -- & -- & 1,0 & -- \\
\hline Callao & 1,0 & $0,7-1,3$ & 0,953 & $0,90,7-1,3$ & 0,683 \\
\hline Trujillo & 1,3 & $0,9-1,8$ & 0,102 & $1,1 \quad 0,8-1,6$ & 0,500 \\
\hline Arequipa & 0,8 & $0,5-1,1$ & 0,167 & $0,8 \quad 0,5-1,2$ & 0,500 \\
\hline Cuzco & 0,9 & $0,6-1,2$ & 0,386 & $0,9 \quad 0,7-1,3$ & 0,500 \\
\hline Huamanga & 1,7 & $1,3-2,3$ & $<0,001$ & $1,8 \quad 1,4-2,4$ & $<0,001$ \\
\hline Maynas & 1,0 & $0,8-1,4$ & 0,795 & $1,0 \quad 0,8-1,4$ & 0,500 \\
\hline
\end{tabular}

*Modelo multivariado ajustado por sexo, edad, grado de instrucción, provincia, consumo excesivo de alcohol en el último mes e ingreso mensual familia, con error estándar robusto

con educación superior universitaria completa en comparación con personas con secundaria incompleta o menos (OR 0,8; IC 95\%: 0,6-1,0; $p=0.030$ ). Por otro lado, existe mayor aceptabilidad del castigo físico en hombres que en mujeres (OR=1,2; IC 95\%: 1,0-1,4; $p=0,020)$. EI hecho de que ambas asociaciones resultaron con un valor $p$ cercano a 0.05 nos obligó a considerarlas como marginales, sin descartar la posibilidad de error tipo I, especialmente en el contexto del tamaño de muestra de 
la magnitud del presente estudio. De manera interesante, sin embargo, nuestro estudio encuentra que las personas residentes de la provincia de Huamanga en la región de Ayacucho revelaron mayor aceptabilidad por el castigo físico que los residentes de las otras localidades (OR 1,8; IC 95\%: 1,4-2,4; $p<0,001)$.

Asimismo, en el modelo ajustado se perdió una asociación significativa entre los grupos de edad y la aceptabilidad del castigo físico. El consumo de alcohol y el ingreso familiar mensual promedio no resultaron asociados en el modelo crudo ni en el ajustado.

Por su parte, el análisis de sensibilidad realizado para la elección de la dicotomización de la variable desenlace no generó resultados diferentes a los obtenidos con la categorización original de la variable Aceptabilidad (i.e., 1= "si"; 0= "no/no seguro"), descrito en la sección de métodos. Por lo tanto, se decidió mantener esta última categorización de la variable dependiente para realizar los análisis reportados en el presente estudio. De igual modo, el análisis de sensibilidad realizado para evaluar si la población menor de 18 años modificaba los estimados, encontró que las estimaciones de OR permanecieron sin variación notable a los OR obtenidos con toda la población de estudio. Finalmente, para los lectores que prefieren el uso del RP para estimar la fuerza de asociación, en nuestro análisis de sensibilidad se encontró una diferencia de solo un decimal entre ambas estimaciones (de un OR de 1,8 a un RP de 1,7).

\section{DISCUSIÓN}

El presente estudio encontró que las personas expuestas a violencia física durante la niñez tienen más riesgo de aceptarla o justificarla durante la adultez. Este hallazgo es congruente con la hipótesis de que el abuso infantil tiene consecuencias a largo plazo, incluyendo la formación de cogniciones que justifican la violencia física como forma de educar a los niños, lo que a su vez hace más probable que el adulto cometa abuso físico hacia los niños a su cargo. Por otra parte, aunque el presente estudio ha encontrado asociación inversa entre el nivel educativo y la aceptabilidad de castigo físico en la crianza de los niños, como se ha mencionado, esta es estadísticamente inestable con valores $p$ cercanos a 0,05 . Esto nos obliga a ser cuidadosos en interpretar estos resultados y esperar que futuros estudios obtengan resultados consistentes antes de presentar conclusiones. Lo mismo se cumple para las diferencias encontradas respecto al sexo. Por último, la mayor aceptabilidad del castigo físico en la provincia de Huamanga, debe ser estudiada explorando si ello podría asociarse con la mayor exposición a la violencia estructural ocurrida en esa parte del país en la década de los 80. De acuerdo con el informe final de la Comisión de la Verdad y Reconciliación (CVR), Ayacucho fue la región con mayor exposición a la violencia, evidenciando el mayor porcentaje $(40 \%)$ de muertos y desaparecidos en el Perú (27). Además, existe evidencia sobre mayor prevalencia de otros tipos de violencia en Ayacucho que en otras regiones del país, como Lima. Por ejemplo, un estudio encontró mayor violencia doméstica en Ayacucho que en Lima. Las diferencias fueron significativas, tanto para la proporción de mujeres víctimas (Ayacucho 19,8\%, Lima 8,4\% p<0,001) como para la proporción de agresores (Ayacucho 12,9\% y Lima $6,5 \%, p<0,001)^{(28)}$.

Entre las limitaciones más resaltantes del presente estudio es que no se pudieron realizar análisis estadísticos para muestras complejas que tome en cuenta las ponderaciones y los niveles de muestro. El no incluir los pesos del muestreo en el análisis puede afectar la exactitud de los estimados, sin embargo, esta diferencia es mínima, de modo que no afectaría la dirección ni la magnitud de las medidas de asociación de forma significativa. Asimismo, debido a que no tomar en cuenta el muestro complejo puede llevar a una mayor probabilidad de cometer error tipo I, el presente estudio se enfoca en los resultados con valores $p<0,001$. Así, el hecho de que, en nuestro estudio, la asociación entre la aceptabilidad del castigo físico y haber sido objeto de violencia física durante la niñez tiene estimados estadísticos precisos, a juzgar por la amplitud del intervalo de confianza al 95\%, y un valor p menor a 0,001 , proporciona confianza respecto a la consistencia del estimado. Similarmente, esto se cumple para la asociación entre residir en Huamanga y la aceptabilidad del castigo físico.

Otra posible limitación del estudio tiene que ver con el sesgo de información que podría surgir de dos circunstancias. La primera tiene que ver con que la exposición se cuantificó en base al recuerdo que los participantes podían tener de eventos ocurridos hace mucho tiempo y en un periodo muy temprano de sus vidas. La segunda circunstancia tiene que ver con que la pregunta de aceptabilidad del castigo físico en niños puede no haber sido entendida con precisión por los participantes. Sin embargo, consideramos que el potencial error de medición derivado de estas dos circunstancias, de existir, no es diferencial entre los grupos en comparación. Específicamente, no hay razones para sospechar que el reporte de victimización física en la niñez en el caso de las personas que aceptan la violencia física en la crianza de niños es diferente de las que no la aceptan. Asimismo, no encontramos razones como para sospechar que aquellas personas que fueron víctimas de violencia física en la niñez puedan entender de manera diferente la pregunta de aceptabilidad que aquellas que 
no fueron víctimas. Este balance de errores de medición, que generalmente diluye la magnitud de la asociación no parece haber impactado en nuestro estudio, a juzgar por la magnitud, precisión y consistencia de la asociación encontrada (expresadas en el valor puntual, intervalo de confianza y valor $\mathrm{p}$, respectivamente)

Entre las fortalezas del presente estudio se incluye que la muestra ha sido seleccionada de la población general, basado en un diseño aleatorizado de selección de participantes en sus hogares, lo cual permite controlar sesgos de selección que dependan o de la exposición o del evento resultado. Así, el muestreo poblacional del presente estudio permite evitar la selección diferenciada de sujetos como sucede cuando el estudio se basa en establecimientos de salud o de atención de víctimas de violencia, donde aquellas víctimas que llegan a ser atendidas pueden ser cualitativamente diferentes a aquellas víctimas que no llegan a buscar ayuda y así resultar encontrando asociaciones espurias (sesgo de Berkson).

Otra fortaleza tiene que ver con la oportunidad que nos proporciona este estudio de establecer temporalidad entre la exposición (haber sufrido violencia física durante la niñez), y el evento resultado (aceptabilidad del castigo físico en la crianza de los niños en el momento actual). Esto permite, aun cuando el presente estudio es de diseño transversal, añadir evidencia consistente con la hipótesis causal de que el antecedente de violencia física incrementa el riesgo de que la persona tenga al llegar a la edad adulta una opinión favorable al castigo físico hacia los niños.

Así, tomando en cuenta las limitaciones y fortalezas del presente estudio, realizado en población general en ciudades peruanas, y considerando lo robusto y preciso del estimado de asociación entre la exposición a la violencia física en la niñez con la aceptabilidad más tarde en la vida del castigo físico como recurso que puede ser usado en la crianza de los niños, proporciona evidencia confiable de que en Perú se cumple lo que se ha encontrado en otras sociedades a pesar de las diferencias socioculturales que puedan existir (24,25).

A partir del presente estudio surgen, sin embargo, nuevas interrogantes acerca de las características de la exposición a la violencia en la infancia y la posterior aceptabilidad del castigo físico. Por ejemplo, sería interesante explorar si el grado, tipo o el tiempo de exposición a la violencia durante la infancia impactan en el nivel de aceptabilidad del castigo físico en la adultez, y en la eventual traducción de esta aceptabilidad en un acto violento hacia los niños que se tiene a cargo.

Finalmente, estos hallazgos proporcionan evidencia de temporalidad, y son consistentes con otros estudios $(24,25)$ y coherentes con la teoría del aprendizaje social ${ }^{(22)}$ y la del procesamiento de información social ${ }^{(23)}$. Ello hace que presten fuerza a la teoría causal que indica que el haber sido expuesto a violencia física durante la niñez incrementa el riesgo de tener cogniciones que aceptan el castigo físico en la crianza de los niños. Sin embargo, evidencia más concluyente de causalidad debe ser generada a partir de diseños experimentales. Por ejemplo, ensayos comunitarios donde se muestre que intervenciones dirigidas a reducir la violencia física en la niñez llevan a reducir la aceptación en los adultos de que en ocasiones el castigo físico es justificado en la crianza de los niños y que a su vez ello se convierta en una reducción real del acto de castigar pueden ser valiosos.

En conclusión, en un contexto como el peruano, el haber recibido castigo físico en la niñez está asociado, quizá causalmente, con el hecho de que la persona tenga cogniciones que acepten o justifiquen el castigo físico en la crianza de los niños, lo cual a su vez se ha visto está asociado con cometer fácticamente abuso contra los niños en el hogar ${ }^{(20,21)}$. De todo esto se desprende, que intervenciones orientadas a disminuir la ocurrencia de maltrato infantil en el hogar, pueden tener el efecto adicional de prevención primaria al evitar que se puedan estar generando futuros perpetradores de este tipo de maltrato.

Conflictos de interés: los autores declaran no tener conflictos de interés.

Fuentes de financiamiento: autofinanciado.

Contribuciones de autoría: $A B$ y FF concibieron la idea del estudio y realizaron el análisis estadístico. $A B$ escribió el primer borrador del manuscrito, MP, FF, GA y AG contribuyeron en la revisión crítica del manuscrito. Todos los autores aprueban la versión final del manuscrito. 


\section{REFERENCIAS BIBLIOGRÁFICAS}

1. Naciones Unidas: Comité de los Derechos del Niño. Convención sobre los Derechos del Niño: Observación general $\mathrm{N}^{\circ} 13$ (2011) Derecho del niño a no ser objeto de ninguna forma de violencia. Nueva York: ONU; 2011.

2. Fondo de las Naciones Unidas para la Infancia. Hidden in Plain Sight: A statistical analysis of violence against children | UNICEF Publications [Internet]. Nueva York: UNICEF; 2014 [citado el 14 de octubre de 2014]. Disponible en: http://www.unicef.org/ publications/index_74865.html

3. Fondo de las Naciones Unidas para la Infancia. Unicef responde: Maltrato Infantil en Chile [Internet]. Nueva York: UNICEF; 2014 [citado el 14 de octubre de 2014]. Disponible en: http://unicef.cl/web/unicef-respondemaltrato-infantil-en-chile/

4. González MR, Trujillo A, Pereda $\mathrm{N}$. Corporal punishment in rural Colombian families: prevalence, family structure and socio-demographic variables. Child Abuse Negl. 2014 May;38(5):909-16. doi: 10.1016/j. chiabu.2013.10.006.

5. Ministerio de la Mujer y Poblaciones Vulnerables. Plan Nacional de Acción por la Infancia y la Adolescencia 20122021 (PNAIA) 2021. Lima: MIMP; 2014.

6. Instituto Nacional de Estadística e Informática. Salud sexual y reproductiva de los varones, 2008. Informe general. Endes Varones. 2008. Lima: INEI; 2010.

7. Children's Bureu, Department of Health \& Human Services. Child Maltreatment 2011. Washington, D. C.: Children's Bureu; 2011.

8. Zolotor AJ. Corporal punishment. Pediatr Clin North Am. 2014 Oct;61(5):971-8. doi: 10.1016/j. pcl.2014.06.003.

9. Organización Mundial de la Salud. Maltrato infantile. Nota descriptive $\mathrm{N}^{\circ} 150$ [Internet]. Ginebra: OMS; 2014 [citado el 13 de abril de 2014]. Disponible en: http://www.who.int/ mediacentre/factsheets/fs150/es/

10. Zielinski DS. Child maltreatment and adult socioeconomic well-being. Child
Abuse Negl. 2009 Oct;33(10):666-78 doi: 10.1016/j.chiabu.2009.09.001.

11. Duncan RD. Childhood Maltreatment and College Drop-Out Rates Implications for Child Abuse Researchers. J Interpers Violence. 2000 Sep 1;15(9):987-95.

12. Lansford JE, Dodge KA, Pettit GS, Bates JE, Crozier J, Kaplow J. A 12-year prospective study of the long-term effects of early child physical maltreatment on psychological, behavioral, and academic problems in adolescence. Arch Pediatr Adolesc Med. 2002 Aug;156(8):824-30.

13. Haskett ME, Smith Scott S, Grant R, Ward CS, Robinson C. Child-related cognitions and affective functioning of physically abusive and comparison parents. Child Abuse Negl. 2003 Jun;27(6):663-86.

14. Bower-Russa M. Attitudes mediate the association between childhood disciplinary history and disciplinary responses. Child Maltreat. 2005 Aug;10(3):272-82.

15. Seng AC, Prinz RJ. Parents who abuse: what are they thinking? Clin Child Fam Psychol Rev. 2008 Dec;11(4):163-75. doi: 10.1007/s10567-008-0035-y.

16. Muller RT, Hunter JE, Stollak G. The intergenerational transmission of corporal punishment: a comparison of social learning and temperament models. Child Abuse Negl. 1995 Nov;19(11):1323-35.

17. Ertem IO, Leventhal JM, Dobbs S. Intergenerational continuity of child physical abuse: how good is the evidence? Lancet. 2000 Sep 2;356(9232):814-9.

18. Lostaunau V, Torrejón C, Becerra 1 , Otero S. Un estudio sobre violencia transgeneracional en madres peruanas: perfil clínico-epidemiológico. Rev Peru Epidemiol. 2012;16(2):91-5.

19. Fiestas F, Rojas R, Gushiken A, Gozzer E. ¿Quién es la víctima y quién el agresor en la violencia física entre parejas? Estudio epidemiológico en siete ciudades del Perú. Rev Peru Med Exp Salud Pública. 2012 Ene-Mar;29(1):44-52.

20. Francis KJ, Wolfe DA. Cognitive and emotional differences between abusive and non-abusive fathers. Child Abuse Negl. 2008 Dec;32(12):1127-37. doi: 10.1016/j.chiabu.2008.05.007.
21. Bailey HN, DeOliveira CA, Wolfe VV, Evans EM, Hartwick C. The impact of childhood maltreatment history on parenting: a comparison of maltreatment types and assessment methods. Child Abuse Negl. 2012 Mar;36(3):236-46. doi: 10.1016/j.chiabu.2011.11.005.

22. Wolfe D, Peter GJ. Emerging strategies in the prevention of domestic violence. Domest Violence Child. 1999;9(3):133-44.

23. Rodriguez CM. Personal contextual characteristics and cognitions: predicting child abuse potential and disciplinary style. J Interpers Violence. 2010 Feb;25(2):315-35. doi: $10.1177 / 0886260509334391$.

24. Orue I, Calvete E. La justificación de la violencia como mediador de la relación entre la exposición a la violencia y la conducta agresiva en infancia. Psicothema. 2012 Feb;24(1):42-7.

25. Calvete E. Justification of violence beliefs and social problem-solving as mediators between maltreatment and behavior problems in adolescents. Span J Psychol. 2007 May;10(1):131-40.

26. Fournier M, de los Rios R, Orpinas $P$, Piquet-Carneiro L. [Multicenter Study on Cultural Attitudes and Norms towards Violence (ACTIVA project): methodology]. Rev Panam Salud Publica. 1999 Apr-May;5(4-5):222-31. [Artículo en Español].

27. Comisión de la Verdad y la Reconciliación. Informe Final de la Comisión de la Verdad y la Reconciliación: Conclusiones Generales. Lima: CVR; 2003.

28. Graham K, Bernards S, Munné M, Wilsnack S. El Brindis Infeliz: el Consumo de Alcohol y la Agresión entre Parejas en las Américas. En: M. Piazza. El alcohol y la agresión física en la pareja en Lima y Ayacucho. p. 189-211.

Correspondencia: Fabián Fiestas

Dirección: Cápac Yupanqui 1400 Jesús María, Lima Perú

Correoelectrónico:fabianfiestas@yahoo.com 